\title{
DETERIORO DEL VALOR DE LOS ACTIVOS MINEROS SEGÚN LAS NORMAS INTERNACIONALES DE CONTABILIDAD Y LA GESTIÓN DE RESULTADOS: EVIDENCIA PARA LAS EMPRESAS MINERAS CHILENAS
}

\section{IMPAIRMENT OF MINING ASSETS ACCORDING TO THE INTERNATIONAL ACCOUNTING STANDARDS AND EARNINGS MANAGEMENT: EVIDENCE FOR CHILEAN MINING}

\author{
Claudia Orellana Fuentes ${ }^{1}$
}

\section{RESUMEN}

El año 2015 estuvo marcado por la caída de los precios de diversos metales, entre ellos el cobre. Chile se caracteriza por ser un país históricamente minero y múltiples han sido los efectos que han sido presagiados en la prensa nacional.

Este estudio tiene por objetivos en primer lugar, verificar los efectos reales de la caída del precio del cobre en los estados financieros de las empresas mineras chilenas, para lo cual se estudió una muestra de memorias anuales obtenidas de 33 empresas mineras. En segundo lugar, constatar si los administradores de las empresas mineras de la muestra usan de la discrecionalidad conferida por las normas internacionales de contabilidad en la aplicación de los test de deterioro de valor de los activos.

Se analiza el impacto de la caída del precio del cobre en los rubros de Inventarios, Propiedades, Plantas y Equipos (PPE) y Activos Intangibles.

Los resultados confirman que sólo 36\% del total de las empresas de la muestra aplicaron algún test de deterioro a sus activos, y que aquellas que presentan un mayor impacto en los Estados Financieros se relacionan con activos deteriorados que se asocian a operaciones mineras en fase de decadencia o dificultades de explotación.

Palabras claves: Minería, Chile, Test de deterioro.

Recepción: 10/11/2017. Aprobación: 22/12/2017.

\section{ABSTRACT}

The year 2015 was marked by a decline in the prices of different metals, including copper. Chile is characterized by being a historically mining country, and multiple effects have been foretold in the national media.

The objective of this research is, firstly, to verify the real effects of the decline of the price of copper in the financial statements of the Chilean mining companies by studying a sample of annual financial statements of 33 mining companies. Secondly, to confirm if the managers of the mining companies of the sample use the discretion provided by the International Accounting Standards in the application of impairment tests to their assets.

1 Pontificia Universidad Católica de Valparaíso, Valparaíso, Chile, e-mail claudia.orellana@pucv.cl 
Specifically, the impact of the decline in the price of copper it's analyzed regarding the carrying amount of Inventories, Property, Plant and Equipment (PP\&E), and Intangible Assets.

The results confirm that only $36 \%$ of the companies of the sample applied an impairment test to their assets, and those that show a greater impact on the Financial Statements are related to mining operations that have exploitation difficulties.

Keywords: Mining Chile, Impairment Test.

\section{INTRODUCCIÓN}

El panorama de la contabilidad en este siglo ha sido marcado por el hito alcanzado por el International Accountant Standards Board (IASB), que ha logrado la aplicación de sus normas en casi todo el mundo ${ }^{2}$. El IASB ha dejado de manifiesto en múltiples ocasiones que las normas internacionales de contabilidad estarían basadas en principios, y que por ello demanda el juicio profesional y ética de las personas que deben aplicarlas. Como consecuencia de esta visión del emisor de normas contables, existen en estas normas espacios de libertad o discrecionalidad en las decisiones asociadas a la contabilidad que pueden ser tomadas por los dirigentes de las empresas. Este margen de maniobra les permitiría, si así lo desearan, sesgar la información contable emitida por la entidad para influir en la toma de decisiones de los usuarios, sin por ello caer en una configuración fraudulenta.

El poder discrecional de los dirigentes en cuanto a la forma y contenido de la información contable no es un tema nuevo en la investigación contable, éste ha sido ampliamente abordado en la literatura contable anterior.

Stolowy \& Breton (2003) proponen una tipología para la clasificación de las diversas manifestaciones asociadas al poder discrecional de los administradores en la aplicación de las normas contables. Estos autores han denominado de forma general a este tipo de actividades como la "gestión de datos contables", siendo la "gestión de resultados o earnings management" ${ }^{\prime \prime}$ una de sus categorías.

Estos autores definen la gestión de datos contables como "la explotación de la discreción dejada a los dirigentes en materia de opciones contable o de reestructuración de las operaciones, con el objetivo de generar una modificación del riesgo de transferencia de riquezas asociadas a la empresa, tales como el riesgo que es percibido en la práctica por el merca- do" (Stolowy \& Breton, 2003, p. 130, traducción libre).

La gestión de datos contables que no responden a conductas fraudulentas, surgiría de las diferencias producidas entre la contabilidad de caja, la cual da cuentas de las operaciones contables solo cuando éstas afectan a los flujos de efectivo, y la contabilidad en base a devengado, que exige el registro contable de las operaciones independientemente que dichas operaciones afecten los flujos de efectivos de la entidad, generando por lo tanto, lo que se conoce en la literatura contable como los accruals. Los accruals son ajustes contables que no afectarán al flujo de efectivo de la entidad sino hasta en periodos futuros, por ejemplo, la depreciación, la amortización y los deterioros de valor de activos.

Revisión de literatura. Diversos autores sugieren que la administración de una empresa utiliza su poder discrecional sobre el deterioro del valor de los activos para de esta forma gestionar sus resultados (Elliott \& Shaw, 1988; Walsh, Craig \& Clarke, 1991; Zucca \& Campbell, 1992; Elliott \& Hanna, 1996; Jordan \& Clark, 2004; Sevin \& Schroeder, 2004).

Hilton \& O'Brien (2009) a través de un estudio de caso a un empresa minera explotadora de nickel demostraron como la administración de esta empresa de forma deliberada y oportunista postergó el reconocimiento de pérdida de valor de una explotación, dejando a la evidencia el alto poder discrecional que permite la norma contable americana y sobre todo, las posibilidad de manipulación asociadas a las técnicas de cálculo del valor en uso a través de los flujos de efectivos futuros asociados al activo en cuestión, lo que para las autoras incorpora altos grados de subjetividad a la contabilidad.

Este estudio tendrá por objetivo explorar el efecto de la caída del precio del cobre en los activos mineros, más particularmente conocer si las empresas chilenas aplicaron los test de deterioro

2 De acuerdo al IASB, de los 143 países analizados en su informe el 93\% exige o autoriza la aplicación de las normas IFRS para las empresas que hacen públicos sus estados financieros. http:/ / www.ifrs.org/Use-around-the-world/Pages / Analysis-of-the-IFRS-jurisdictional-profiles.aspx

3 Se consideran dentro de la gestión de datos contables: la "gestión de resultados (earnings management), el alisado del resultado (income smoothing), limpieza de cuentas (big bath accounting), maquillage de cuentas (window dressing) y la contabilidad creativa (creative accounting)" (Stolowy \& Breton, 2003, p. 126). 
a sus activos mineros al 31 de diciembre 2015, y establecer la magnitud de dichos ajustes, los efectos en los activos y los resultados del periodo.

La actividad minera y el precio del cobre. La actividad minera en Chile, y más particularmente, la explotación del cobre ha sido y sigue ocupando un lugar destacado dentro de la economía del país, con una participación del 10,8\% en el PIB nacional además de una participación del $50 \%$ en el total de las exportaciones del país para el año 2014 (Cochilco, 2015).

El impacto que tiene la caída del precio del cobre en los Estados Financieros de las empresas mineras chilenas, puede ser medido y registrado a través de la aplicación de diversas normas contables. Se pueden distinguir, los efectos de la caída del precio del cobre en activos corrientes, como los inventarios, y los efectos en activos no corrientes, como las PPE, los activos intangibles $\mathrm{y}$ las inversiones en sociedades mineras.

La norma NIC 2 "Inventarios", exige a las entidades valorizar sus inventarios al menor entre el valor libros de éstos y su Valor Neto Realizable (VNR). El VNR se estima en base al valor de mercado de dichos inventarios. De acuerdo a esta norma, no existiría discreción de la administración ya que es una exigencia realizar dicha comparación a cada fecha de reporte.

En cuanto a los activos no corrientes, las
PPE, los activos intangibles e inversiones en sociedades mineras, las normas contables buscan determinar si dichos activos podrían generar suficientes beneficios futuros para cubrir sus valores libros.

Centrándonos, en las PPE y los activos intangibles, ya sea la norma IFRS 6 "Exploración y Evaluación de Recursos Minerales" o la norma NIC 36 "Deterioro del valor de los Activos" exigen la revisión de estos activos cuando existen indicios de pérdida de valor, siendo la disminución del valor del mineral uno de estos indicios de deterioro. Cabe destacar, que la aplicación de los test de deterioro depende del juicio de los administradores, ellos deben evaluar si existe evidencia suficiente para aplicar un test de deterioro a algún activo. De lo anterior se desprende que el nivel de discreción es importante en la aplicación de las normas asociadas al deterioro de valor de activos no corrientes en la industria minera.

Precio del cobre y la aplicación del Test de Deterioro. En el año 2015, luego de un periodo sostenido de precios a niveles sobre los 3 dólares la libra, el precio se desploma, llegando a niveles que bordean los 2 dólares americanos la libra. Como consecuencia de la caída del cobre, se hizo común ver empresas mineras que reducían sus actividades, o simplemente cerraban ${ }^{4}$, paralizando masivamente inversiones nuevas.

Gráfico 1. Precio del cobre de la bolsa London Metal Exchange (2010-2016).

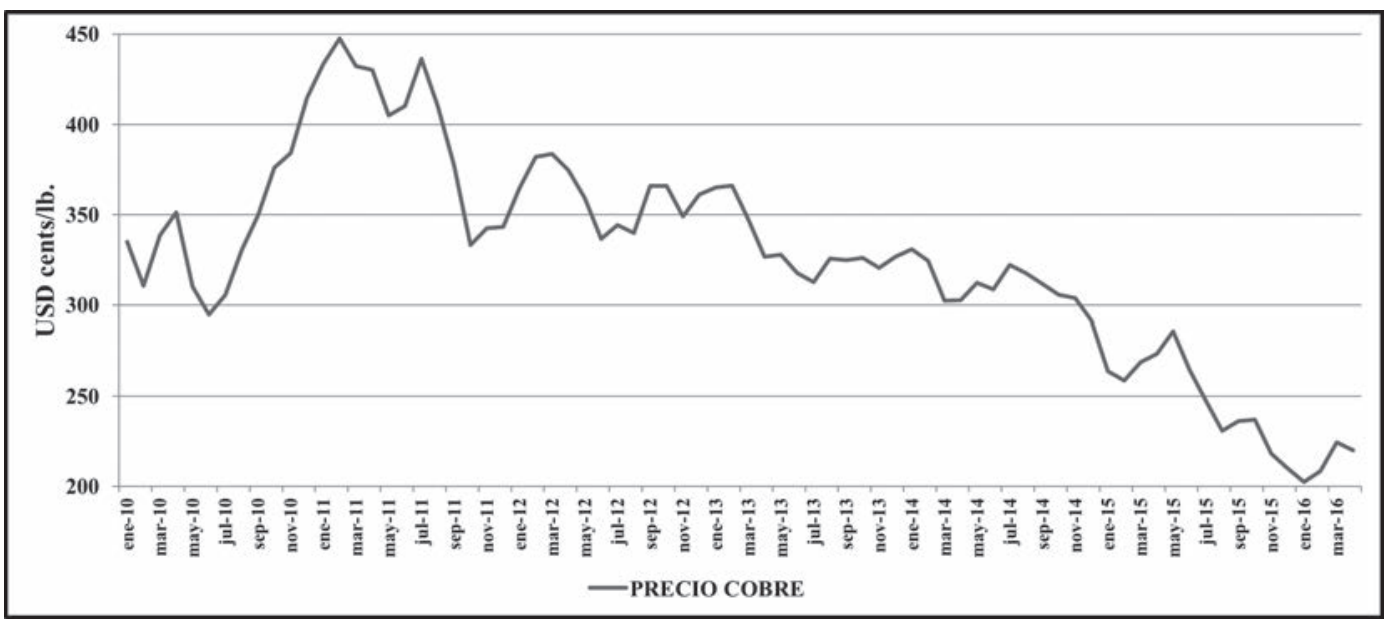

Fuente: Elaboración propia en base a información obtenida de www.cochilco.cl.

4 Ver "Fin del súper ciclo lleva a la quiebra a 18 productoras de cobre, hierro y carbón" Disponible en: https://www.df.cl/noticias/empresas/mineria/ fin-del-super-ciclo-lleva-a-la-quiebra-a-18-productoras-de-cobre-hierro/2016-03-29/201343.html (10/04/2016). Ver "Pequeños productores de cobre de Antofagasta advierten con movilizaciones" Disponible en: http://www.mch.cl/2015/12/09/pequenos-productores-de-cobre-de-antofagasta-advierten-con-movilizaciones/ (10/04/2016). Ver "Consejo de Sonami asume escenario aún más duro y anticipa cierre de decenas de faenas" Disponible en: http://www.pulso.cl/noticia/empresa---mercado/empresa/2015/11/11-74904-9-consejo-de-sonami-asume-escenario-aun-mas-duro-y-anticipacierre-de-decenas-de.shtml $(10 / 04 / 2016)$. 
En cuanto a los indicios de deterioro, la norma IFRS $6 \S 20$ establece los indicios específicos asociados a activos para la exploración y evaluación, y en la letra d) establece que si: "existen datos suficientes para indicar que, aunque es probable que se produzca un desarrollo en un área determinada, resulta improbable que el importe en libros del activo para exploración y evaluación pueda ser recuperado por completo a través del desarrollo exitoso o a través de su venta". En efecto la caída del precio del mineral, tendría un efecto directo en los flujos que puedan ser recuperados de un proyecto minero en evaluación, formando parte de uno de los indicios de deterioro que los dirigentes deberían considerar para evaluar la existencia de una pérdida de valor en estos activos.

En cuanto a la NIC $36 \S 9$ establece que "la entidad evaluará, en cada fecha de cierre del balance, si existe algún indicio de deterioro del valor de algún activo. Si existiera tal indicio, la entidad estimará el importe recuperable del activo".

Es un indicio de deterioro cuando"(a) durante el ejercicio, el valor de mercado del activo ha disminuido significativamente más que lo que cabría esperar como consecuencia del paso del tiempo o de su uso normal [...] (g) Se dispone de evidencia procedente de informes internos, que indica que el rendimiento económico del activo es, o va a ser, peor que el esperado" (NIC $36 \S 12$ ).

Considerando ambas normativas, los dirigentes de las entidades mineras del mundo, y particularmente las empresas mineras chilenas que explotan cobre, frente al indicio de deterioro asociado a la caída del precio del cobre y las expectativas de la industria sobre precios bajos en al menos los próximos tres años ${ }^{5}$, deberían aplicar los test deterioro correspondientes a sus activos mineros en los Estados Financieros presentados al 31 de diciembre 2015.

Este estudio busca entregar evidencia de las consecuencias asociadas a la baja del precio del cobre, con el fin de verificar los niveles de discreción de los dirigentes de las empresas mineras chilenas.

\section{METODOLOGÍA}

Los activos mineros que serán revisados serán: los inventarios, las PPE y los activos intangibles. Para tal efecto se utilizará una fuente de información pública, las memorias disponibles en la página web de la Superintendencia de Valores y Seguros ${ }^{6}$ (SVS). La muestra de empresas mineras se asocia a aquellas empresas mineras que están obligadas a publicar sus Estados Financieros como resultado de las exigencias legales asociadas a ciertos beneficios fiscales, luego de la emisión de Ley 20.026 que grava a la industria minera con un impuesto específico. Cabe destacar, que en Chile, sólo las entidades que se han financiado en el mercado de valores están obligadas a hacer públicos sus Estados Financieros, considerando que el fuerte de las empresas mineras establecidas en Chile no recurre al mercado local para financiar sus actividades, la mayor parte de ellas son financiadas a través de los aportes directos de sus casas matrices que representan a los grandes grupos multinacionales de la industria extractiva.

Es por ello, que tan sólo dos empresas mineras financian parte de sus actividades recurriendo al mercado local y por lo tanto deben cumplir los requisitos de emisión de información financiera y contable a la SVS, lo cual muestra que las exigencias de la Ley 20.026 permiten obtener las memorias anuales auditadas de las empresas mineras chilenas que en condiciones normales quedarían solo en el ámbito privado.

En base a la información pública se realizará un estudio de los ítems asociados a los inventarios, las propiedades, plantas y equipos y los activos intangibles, las notas explicativas y otras informaciones asociadas a los posibles deterioros de valor efectuados y registrados en el año 2015.

Esta información permitirá establecer si los dirigentes de estas empresas mineras consideraron el indicio de deterioro "caída del precio del cobre" para realizar los test de deterioro a los activos mineros correspondientes, y de esta manera poder visualizar el grado de discrecionalidad con que cuentan los dirigentes de estas empresas mineras chilenas asociados a este tema.

\footnotetext{
5 Pizarro, C.; González, F. \& Álvarez, G. (2015, 11, 25). Diego Hernández y caída del cobre: "Ya se puede decir que es una crisis". [on line] La Tercera. Disponible en: http://www.latercera.com/noticia/negocios/2015/11/655-657405-9-diego-hernandez-y-caida-del-cobre-ya-se-puede-decirque-es-una-crisis.shtml $(10 / 04 / 2016)$. González, F. $(2015,11,18)$. Esperamos tres años complejos. [on line] La Tercera. Disponible en: http://diario. latercera.com/2015/11/18/01/contenido/negocios/10-202961-9-esperamos-tres-anos-complejos.shtml (10/04/2016). Namur, P. (2015, 12, 02). FMI: impacto de caída del cobre en Chile se sentirá por 10 años. [on line] El Pulso. Disponible en: http://www.pulso.cl/noticia/empresa---mercado/ internacional/2015/12/72-75153-9-fmi-impacto-de-caida-del-cobre-en-chile-se-sentira-por-10-anos.shtml (10/04/2016).

6 Institución chilena encargada de "velar por la transparencia de los mercados que supervisa, mediante la oportuna y amplia difusión de la información pública que mantiene y, colaborar en el conocimiento y educación de inversionistas, asegurados y público en general. Todos ellos, elementos esenciales para el desarrollo y correcto funcionamiento de dichos mercados" www.svs.cl.
} 
La muestra: El grupo de empresas que deben publicar sus estados financieros se conforma de 33 empresas mineras. Sólo 14 de estas empresas cuentan con capitales chilenos y una de ellas es de propiedad del Estado. Se pueden encontrar inversionistas de filiales asociadas a los grandes grupos mineros mundiales, tal como Barrick Gold, BHP Billiton, Freeport McMoran, Teck, Angloamerican, Glencore, Mitsui, Sumimoto, Marubeni, Yamana Gold, Far West, Kinross Gold, entre otros, además de algunos grupos chilenos como Antofagasta Minerals de la familia Luksic.

De esta muestra, 25 empresas explotan cobre o algún subproducto del cobre, 4 de ellas explotan nitratos y el resto explotan metales preciosos como el oro y la plata.

Las memorias anuales presentadas por estas empresas para el año 2015 muestran que estas empresas detienen MMUS\$ 105.597 en activos totales y los resultados del ejercicio totalizan pérdidas por MMUS\$1.186. El 70\% de las empresas de la muestran presentaron pérdidas para el año 2015, lo que confirma los efectos inmediatos de la baja de los precios de los metales, tales como el cobre, el oro, la plata, el molibdeno y el hierro.

\section{RESULTADOS}

Todas las empresas de la muestra han sido auditadas por grandes grupos mundiales de auditoría: 9 empresas por Deloitte, 3 empresas por Ernest \& Young, 6 empresas por KMPG, 14 empresas por PriceWaterhouseCoopers (PWC) y 1 empresa que no específica la empresa auditora.

La totalidad de empresas de la muestra declararon el uso de las normas contables emitidas por el IASB, y establecen claramente en las notas explicativas las políticas y criterios contables para el registro de los inventarios, PPE, activos intangibles, actividades de prospección y exploración, las exigencias de deterioro de valor de los activos y el reverso del deterioro de valor, todo esto conforme a las normas contables vigentes: NIC 2 Inventarios, NIC 16 Propiedades, Planta y Equipos, NIIF 6 Exploración y Evaluación de Recursos Minerales, NIC 38 Activos Intangibles y NIC 36 Deterioro del valor de los activos.

De las 33 empresas de la muestra, solo $36 \%$ de ellas informaron en sus estados financieros pérdidas por la aplicación de algún test de deterioro en algún activo clasificado ya sea en PPE, Activos Intangibles o Inventarios de acuerdo a la Tabla 1.

Tabla 1. Efecto del deterioro del valor en el total de activos.

\begin{tabular}{|c|c|c|c|c|c|c|c|}
\hline \multirow{2}{*}{\multicolumn{2}{|c|}{ Empresas mineras }} & \multicolumn{4}{|c|}{ Efecto en activos deteriorados } & \multirow{2}{*}{$\begin{array}{c}\text { Total } \\
\text { activos }\end{array}$} & \multirow{2}{*}{$\begin{array}{c}\text { Disminución } \\
\text { del total } \\
\text { activos }\end{array}$} \\
\hline & & PPE & Intangibles & Inventarios & Total & & \\
\hline 1 & Anglo American Sur & 274.000 & 0 & 0 & 274.000 & 5.169 .702 & $-5,03 \%$ \\
\hline 2 & M. Maricunga S.A. & 23.586 & 0 & 30.597 & 54.183 & 610.231 & $-8,16 \%$ \\
\hline 3 & M. Nevada & 503.909 & 0 & 0 & 503.909 & 957.134 & $-34,49 \%$ \\
\hline 4 & M. Quebrada Blanca S.A. & 0 & 0 & 32.110 & 32.110 & 1.070 .563 & $-2,91 \%$ \\
\hline 5 & M. Cerro Colorado & 132.966 & 0 & 387 & 133.353 & 2.601 .635 & $-4,88 \%$ \\
\hline 6 & M. Teck Carmen de Andacollo & 0 & 0 & 2.268 & 2.268 & 801.351 & $-0,28 \%$ \\
\hline 7 & M. Escondida & 78.733 & 0 & 51.630 & 130.363 & 15.882 .175 & $-0,81 \%$ \\
\hline 8 & M. Florida Ltda & 129.687 & 0 & 0 & 129.687 & 304.664 & $-29,86 \%$ \\
\hline 9 & M. CODELCO & 364.577 & 0 & 84.527 & 449.104 & 33.443 .843 & $-1,33 \%$ \\
\hline 10 & SCM M. Lumina Cooper Chile & 709.554 & 12.518 & 0 & 722.072 & 4.564 .447 & $-13,66 \%$ \\
\hline 11 & M. Sierra Gorda SCM & 895.300 & 38.600 & 0 & 933.900 & 5.980 .400 & $-13,51 \%$ \\
\hline 12 & SCM El Abra & 0 & 0 & 71.090 & 71.090 & 1.664 .417 & $-4,10 \%$ \\
\hline & TOTALES EN MUSS & 3.112 .312 & 51.118 & 272.609 & \begin{tabular}{|l|}
3.436 .039 \\
\end{tabular} & 73.050 .563 & \\
\hline
\end{tabular}

Fuente: Elaboración propia en base de las Memorias Anuales 2015 de las empresa de la muestra. 
Las empresas mineras que presentan mayor impacto del deterioro sobre el total de activos son: la Minera Nevada, la Minera Florida y las Mineras Lumina y Sierra Gorda.

La Minera Nevada fue creada para llevar a cabo un proyecto que ha sido muy polémico en Chile en razón a las consecuencias ambientales de su explotación que requiere la intervención de glaciares cordilleranos milenarios. Dicho proyecto llamado Pascua-Lama se encuentra ubicado en la zona limítrofe entre Chile y Argentina. En consideración a la paralización actual de dichas faenas, así como la caída de los precios de largo plazo de la plata fueron considerados los indicios de deterioro de valor por la administración de la Minera Nevada, reflejando deterioro de valor tanto para el año 2014 como para el año 2015.

La Minera Florida por su parte, informa en la memoria anual que "al 31 de diciembre de 2015, el Grupo registró un deterioro a los activos no corrien- tes, totalizando MUS\$129.687, derivado principalmente por la revisión a la baja de los precios de los metales incluyendo el precio de largo plazo por onza de $\$ 1,300$ a $\$ 1,250$. Esto impactó negativamente los flujos futuros de caja estimados y la visión actualizada de los valores por sobre las reservas de mineral y los recursos minerales" (Memoria Minera Florida 2015, p. 17).

Tanto la empresa minera Lumina como la minera Sierra Gorda no entregan información en las notas explicativas sobre los indicios de deterioro de valor que fueron considerados, sin embargo por la prensa especializada se pueden conocer las dificultades asociadas a la puesta en marcha de las explotaciones, en un contexto complejo de caída de precio de minerales, como el molibdeno en el caso de la minera Sierra Gor$\mathrm{da}^{7}$.

En cuanto al efecto del deterioro en los resultados de las empresas mineras, dicho efecto se puede observar en la Tabla 2.

Tabla 2. Efecto del deterioro del valor en los resultados de las empresas de la muestra.

\begin{tabular}{|r|l|r|r|r|}
\hline \multicolumn{2}{|c|}{ Empresas mineras } & $\begin{array}{c}\text { Efecto del deterioro } \\
\text { en resultado }\end{array}$ & $\begin{array}{c}\text { Resultado } \\
\text { periodo }\end{array}$ & $\begin{array}{c}\text { Efecto del } \\
\text { deterioro } \\
\text { en el } \\
\text { resultado }\end{array}$ \\
\hline $\mathbf{1}$ & Anglo American Sur & -270.000 & -109.250 & $-168 \%$ \\
\hline $\mathbf{2}$ & M. Maricunga S.A. & -54.183 & -30.989 & $-234 \%$ \\
\hline $\mathbf{3}$ & M. Nevada & -494.792 & -619.227 & $-398 \%$ \\
\hline $\mathbf{4}$ & M. Quebrada Blanca S.A. & -32.110 & -69.829 & $-85 \%$ \\
\hline $\mathbf{5}$ & M. Cerro Colorado & -133.353 & -213.379 & $-167 \%$ \\
\hline $\mathbf{6}$ & M. Teck Carmen de Andacollo & -2.268 & -11.245 & $-25 \%$ \\
\hline $\mathbf{7}$ & M. Escondida & -130.363 & 1.071 .770 & $-11 \%$ \\
\hline $\mathbf{8}$ & M. Florida Ltda & -129.687 & -147.239 & $-739 \%$ \\
\hline $\mathbf{9}$ & M. CODELCO & -449.104 & -2.328 & $-101 \%$ \\
\hline $\mathbf{1 0}$ & SCM M. Lumina Cooper Chile & -722.073 & -951.235 & $-315 \%$ \\
\hline $\mathbf{1 1}$ & M. Sierra Gorda SCM & -933.900 & -926.600 & $-12793 \%$ \\
\hline $\mathbf{1 2}$ & SCM El Abra & -71.090 & -7.337 & $-112 \%$ \\
\hline & & & & \\
\hline & TOTALES EN MUS\$ & -3.422 .923 & $-\mathbf{2 . 0 1 6 . 8 8 8}$ & \\
\hline
\end{tabular}

Fuente: Elaboración propia en base de las Memorias Anuales 2015 de las empresa de la muestra.

\footnotetext{
7 Vargas, H. (2016, 01, 11). Altos costos y retrasos marcan puesta en marcha de minas Sierra Gorda y Caserones. [on line] Diario Financiero. Disponible en: https:// www.df.cl/noticias/empresas/mineria/altos-costos-y-retrasos-marcan-puesta-en-marcha-de-minas-sierra-gorda-y/2016-01-08/202910. html $(23 / 06 / 2016)$.
} 
Se puede observar de los datos de la Tabla 2 que solo una empresa de la muestra que informaron el registro de algún deterioro de activos para el año 2015 presentó ganancias en el periodo, la empresa minera Escondida, otras 5 empresas hubiesen presentado resultados positivos sin el registro contable del deterioro del valor de sus activos, estas empresas mineras son: Anglo American Sur, Maricunga S.A., Codelco, Sierra Goda SCM y SCM El abra.

El registro del deterioro de valor en los resultados de las 12 empresas que aplicaron deterioro de valor, ha hecho disminuir los resultados de dichas empresas entre un $11 \%$ hasta en un $12.793 \%$. Por lo cual podemos concluir que el efecto en resultado es un efecto significativo.

El deterioro de valor de las PPE reconocido por la empresa estatal Codelco, sin bien es cierto que en términos relativos el impacto es reducido con respecto al total de activos, ésta pérdida tuvo un impacto importante el resultado del periodo. Esto conllevó a un revuelo mediático en el país, debido a la presentación de pérdidas en el ejercicio 2015, considerando que los resultados de esta empresa minera son en gran parte traspasados al Estado chileno vía dividendos. De acuerdo a las notas explicativas presentadas en la memoria anual 2015, el deterioro de valor se asocia a las unidades generadoras de efectivo División Salvador y División Ventanas. Las variables que afectaron dichas pérdidas fueron "el precio del cobre, costos de tratamiento y refinación, tipos de cambio y tasas de descuento" (Memoria Codelco 2015, p. 225), utilizando una tasa de descuento al $8.5 \%$ para la determinación del valor en uso.

Como un análisis adicional, cabe destacar el caso de la Minera Zaldivar dedicada a la explotación de cobre, controlada por Antofagasta Minerals el cual es un importante grupo económico chileno. Dicha empresa fue auditada por la PWC, la cual en su informe del auditor independiente se expresa una opinión con salvedades. Las salvedades de la PWC tienen relación con los "inventarios en pilas de lixiviación por un valor de US\$425.574.617 (...) la Administración está llevando a cabo una revisión de los valores recuperables de dichos activos con el propósito de determinar si existen ajustes por deterioro que debieran registrarse en el estado de situación financiera al 31 de diciembre de 2015. Se espera que tal revisión sea completada durante 2016. En consecuencia, no hemos podido obtener suficiente evidencia de auditoría sobre la recuperabilidad de dichos activos o sobre la necesidad de realizar ajuste alguno a su valor en libros" (Memoria minera Zaldivar 2015, p. 19).

Si bien es cierto, una opinión con salvedad no impide al auditor entregar su opinión profesional, esto es considerada un mal antecedente para cualquier empresa que presente estados financiero auditados. Por otro lado, es interesante destacar la razón de la salvedad, la cual tiene relación directa con este estudio, y confirma la aplicación obligatoria de la NIC 2 en comparación con la NIC 36 o las NIIF 6 las cuales tienen un grado de discreción más importante otorgado a la administración de las empresas.

Finalmente, dos empresas mineras de la muestra, teniendo indicios de deterioro tales como la liquidación de la empresa y cierre temporal de las faenas, no aplicaron test de deterioro a sus activos para el año 2015, estas son las Minera Michilla y la Minera Santo Domingo.

\section{DISCUSIÓN Y CONCLUSIONES}

La evidencia obtenida en este estudio exploratorio sobre las memorias anuales del año 2015 de una muestra de 33 empresas mineras chilenas, no hace más que confirmar el alto grado de discreción con que cuentan los administradores de estas empresas para la determinación de la existencia de indicios de deterioro para realizar los test de deterioro según la norma contable internacional a sus respectivos activos mineros, ya sean estos, inventarios, propiedades plantes y equipos o activos intangibles.

Del total de empresas de la muestra solo el $36 \%$ de ellas aplicaron algún test de deterioro a sus activos PPE, intangibles o inventarios, con efectos en resultados, frente a un indicio de deterioro objetivo obtenido del mercado, es decir, los precios de los metales.

Intentando responder a la causa de esta situación, expusimos los casos de las mineras en donde el impacto del deterioro de valor de los activos había sido más relevante. Todas estas mineras ya presentaban explotaciones con problemas arrastrados de periodos anteriores, siendo la caída del precio del metal un simple detonador de un test de deterioro inevitable.

Lo anterior plantea el objetivo y espíritu de la norma contable y la aplicación de los test de deterioro, si ésta realmente busca su aplicación en los casos en que efectivamente las pérdidas en los activos son casi definitivas, o si por el contrario intenta visualizar anticipadamente una pérdida más probable que improbable, aplicando con ello un criterio conservador.

El caso de la minera Zaldívar que presenta una opinión con salvedad de su auditor por no deteriorar el valor de los inventarios, podría ser 
considerado una evidencia a cerca de la posible reticencia de aplicar estas exigencias contables cuando los resultados del periodo tienden a ser negativos, o simplemente las dificultades reales de estimar lo valores recuperables cuando se refiere a productos en proceso y de los cuales no es posible encontrar evidencia clara de su valor razonable.

Una vía de investigación futura podría ser aquella en donde se puedan estudiar las memorias de las empresas mineras a lo largo de un periodo de 5 o 10 años consecutivos con el fin de detectar con evidencia más sólida, la existencia de la discrecionalidad de los administradores asociada a la consideración de los indicios de deterioro y la aplicación misma de los test de deterioro en los diversos activos mineros afectados por la variación de los precios de metales en el mercado.

\section{REFERENCIAS BIBLIOGRÁFICAS}

Andrews, R. (2012). 2nd Annual International Conference on Accounting and Finance (AF 2012) Fair Value, earnings management and asset impairment: The impact of a change in the regulatory environment. Procedia Economics and Finance, 2, 16-25.

Elliott, J. \& Hanna, J. (1996). Repeated accounting write-offs and the information content of earnings. Journal of Accounting Research, 34, 135155.

Elliott, J., \& Shaw, W. (1988). Write-Offs as Accounting Procedures to Manage Perceptions. Journal of Accounting Research, 26, 91-119.

Hilton, A. \& O'Brien, P. (2009). Inco Ltd.: Market Value, Fair Value, and Management Discretion. Journal of Accounting Research, 47 (1), 179-211.

Jordan, C. \& Clark, S. (2004). Big bath earnings management: the case of goodwill impairment under SFAS No. 142. Journal of Applied Business Research, 20 (2), 63-70.

Rees, L.; Gill, S. \& Gore, R. (1996). An Investigation of Asset Write-Downs and Concurrent Abnormal Accruals. Journal of Accounting Research, 34, 157-169.

Sevin, S. \& Schroeder, R. (2004). Earnings management: evidence from SFAS No. 142 reporting. Managerial Auditing Journal, 20 (1), 47-54.
Stolowy, H. \& Breton, G. (2003). La gestion des données comptables : une revue de la littérature. Comptabilité - Contrôle - Audit, 1 (9), 125-151.

Cochilco (2015). Anuario de estadísticas del cobre y otros minerales 1995-2014. Santiago de Chile: Cochilco.

U.S. Geological Survey (2015). Mineral Commodity Summaries 2015.Virginia: USGS.

Walsh, P., Craig, R. \& Clarke, F. (1991). Big Bath Accounting Using Extraordinary Items Adjustments: Australian Empirical Evidence. Journal of Business Finance \& Accounting, 18 (2), 173189.

Zucca, L. \& Campbell, D (1992). A Closer Look at Discretionary Writedowns of Impaired Assets. Accounting Horizons. 30-41.

Memorias Anuales 2015 de las empresas de la muestra disponibles en www.svs.cl 
Anexo 1. Empresas mineras que operan en Chile que conforman la muestra

\begin{tabular}{|c|c|c|}
\hline & Empresa & giro \\
\hline 1 & Algorta Norte S.A. & nitratos y yodo \\
\hline 2 & Anglo American Sur & cobre \\
\hline 3 & Cia. Contractual Minera Encuentro & cobre \\
\hline 4 & Minera Casale & cobre/oro \\
\hline 5 & Mantos de oro & oro/plata \\
\hline 6 & Minera Maricunga S.A. & oro \\
\hline 7 & Minera Nevada & oro/plata/cobre \\
\hline 8 & Minera Quebrada Blanca S.A. & cobre \\
\hline 9 & Minera Zaldivar & cobre \\
\hline 10 & Cia. Contractual minera calendaria & cobre, oro \\
\hline 11 & Minera Cerro Colorado & cobre \\
\hline 12 & Minera Doña Inés de Collahuasi SCM & cobre, molibdeno \\
\hline 13 & Minera Lomas Bayas & cobre \\
\hline 14 & Minera Teck Carmen de Andacollo & cobre \\
\hline 15 & Mantos cooper S.A. & cobre \\
\hline 16 & Minera Antucoya & cobre \\
\hline 17 & Minera Centinela & cobre \\
\hline 18 & Minera Escondida & cobre \\
\hline 19 & Minera Florida Ltda & oro, plata, zinc \\
\hline 20 & Minera Los pelambres & cobre \\
\hline 21 & Minera Meridian Lda. & oro/plata \\
\hline 22 & Minera Michilla (en liquidación) & cobre \\
\hline 23 & Minera Santo Domingo SCM & cobre/hierro \\
\hline 24 & Minera CODELCO & cobre \\
\hline 25 & Minera Spence S.A. & cobre \\
\hline 26 & SCM Minera Lumina Cooper Chile & cobre \\
\hline 27 & Minera Sierra Gorda SCM & cobre, molibdeno \\
\hline 28 & SCM Atacama Minerals Chile & nitratos y yodo \\
\hline 29 & SCM El Abra & cobre \\
\hline 30 & SCM El Morro & oro, cobre \\
\hline 31 & Sociedad Punta de Cobre SA & cobre \\
\hline 32 & SQM Nitratos SA & Nitratos \\
\hline 33 & SQM Salar SA & Nitratos \\
\hline
\end{tabular}


